\title{
CONSTRUCTION OF A COMPLETE SET OF SOLUTIONS OF A IINEAR PARTIAL DIFFERENTIAL EQUATION IN TWO VARIABLES, BY USE OF PUNCH CARD MACHINES*
}

\author{
BY \\ STEFAN BERGMAN** \\ Brown University
}

1. Introduction. Many problems in Engineering and Physics lead to the determination of a function $U$ satisfying the partial differential equation

$$
\mathrm{L}\left(U^{\prime}\right) \equiv \frac{1}{4}\left(\frac{\partial^{2} U}{\partial x^{2}}+\frac{\partial^{2} U}{\partial y^{2}}\right)+\frac{1}{2} \alpha(x, y) \frac{\partial l^{\prime}}{\partial x}+\frac{1}{2} \beta(x, y) \frac{\partial L^{\prime}}{\partial y}+\gamma(x, y) U^{\prime}=0
$$

in a domain $D$, which function assumes certain prescribed values on the boundary of $D$. Here $\alpha, \beta, \gamma$ are polynomials in $x, y$ (or can be approximated by such polynomials).

Recently a procedure, "the method of particular solutions," has been developed for the solution of problems of this kind [2]. $\dagger$ The idea of this method is to determine at first a "complete" set of particular solutions of (1.1), i.e., a set of functions each of which satisfies (1.1); this set [denoted by $\mathrm{p}_{\nu}(x, y),(\nu=1,2, \cdots)$ ], is chosen in such manner as to possess the property that every solution $U$ can be approximated in any simply connected domain by a conveniently chosen finite combination

$$
\sum_{\nu=1}^{N} a_{\nu} \mathrm{p}_{\nu}(x, y)
$$

of the above particular solutions. In the case of the Laplace equation $\left(\partial^{2} U / \partial x^{2}\right)$ $+\left(\partial^{2} U / \partial y^{2}\right)=0$, such a set can be obtained by taking the real and imaginary parts of the powers $(x+\mathrm{i} y)^{\nu-1},(\nu=1,2, \cdots)$; i.e., $\mathrm{p}_{1}=1, \mathrm{p}_{2}=x, \mathrm{p}_{3}=y, \mathrm{p}_{4}=x^{2}-y^{2}, \mathrm{p}_{5}=2 x y$, etc. In the following, a procedure will be described for finding analogous solutions for any equation of the form (1.1). The second step of the method consists in indicating a rule for determining the $a_{\nu}$ so that (1.2) assumes on the boundary of $D$ values which approximate the prescribed values of $U$. In order to apply the method of particular solutions to an actual problem and obtain numerical results of interest to an engineer or physicist, it is frequently necessary to carry out lengthy computations. These computations can as a rule be performed most efficiently by the use of special computing devices, such as punch card machines. Before the computations can be carried out on such machines, however, it is necessary to organize the computations so that they can be given to the operators of the machines. This organization is often a problem in itself, as in the case of the example given below.

In the present paper there is described a working procedure for computing the above set of particular solutions, and for carrying out the associated numerical computations by the use of punch card machines.

* Received Oct. 11, 1945.

** Now at Harvard University.

$\dagger$ Numbers in brackets refer to references at the end of this paper. 
2. Notation. In the following, it is convenient to use the complex variables $z$ and $\bar{z}$ instead of $x$ and $y$. We have $z=x+\mathrm{i} y, \bar{z}=x-\mathrm{i} y$, where $x$ and $y$ are cartesian coordinates in the plane. We then have

$$
\begin{gathered}
U_{z}=\frac{\partial U}{\partial z}=\frac{1}{2}\left(\frac{\partial U}{\partial x}-\mathrm{i} \frac{\partial U}{\partial y}\right), \quad l_{i}=\frac{\partial U}{\partial \bar{z}}=\frac{1}{2}\left(\frac{\partial U}{\partial x}+\mathrm{i} \frac{\partial U}{\partial y}\right), \\
\iota_{z i}=\frac{1}{4} \Delta U=\frac{1}{4}\left(\frac{\partial^{2} U^{\prime}}{\partial x^{2}}+\frac{\partial^{2} U}{\partial y^{2}}\right) .
\end{gathered}
$$

In terms of $z$ and $\bar{z} \mathrm{Eq}$. (1.1) then assumes the form

$$
\ell_{z i}+. U_{z}+B U_{i}+C U=0,
$$

where $2 A=\alpha+\mathrm{i} \beta, 2 B=\alpha-\mathrm{i} \beta, C=\gamma$.

REMark. In the above case, $B=\bar{A}$. However, in the following we derive formulas without making this assumption and we note that in general (2.1) is equivalent to a system of two equations, one corresponding to the real part and one to the imaginary part.

3. Some previous results on particular solutions. The method to be described in the following is based on the following theorem, proved in [2] p. 542.

TheоRem. For every partial differential equation (2.1) there exists a function

$$
\mathrm{E}(z, \bar{z}, t)=\exp \left[-\int_{0}^{\bar{z}} A d \bar{z}\right]\left[1+\sum_{p=1}^{\infty} t^{2 p_{z}} Q^{(p)}(z, \bar{z})\right]
$$

such that the functions*

$$
\left.\begin{array}{l}
\mathrm{p}_{2 v+1}(z, \bar{z})=\operatorname{Re}\left[z^{\nu} \int_{-1}^{1} \mathrm{E}(z, \bar{z}, t)\left(1-t^{2}\right)^{\nu-1 / 2} d t\right], \\
\mathrm{p}_{2 v+2}(z, \bar{z})=\operatorname{Im}\left[z^{\nu} \int_{-1}^{1} \mathrm{E}(z, \bar{z}, t)\left(1-t^{2}\right)^{\nu-1 / 2} d t\right],
\end{array}\right\}
$$

form the required complete set of particular solutions,** i.e., a set possessing the properties

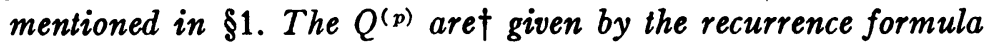

* As has been proved in [1], the series (3.1) converges for $|x|<\infty,|y|<\infty,|t| \leqq 1$ and therefore the functions $p_{\nu}$ are entire functions. We note that they are independent of the domain for which the boundary value problem is considered.

In the N.A.C.A. Technical Notes Nos. 972, 973, 1018 and 1096 the functions $Q^{(p)}(z, \bar{z})$ have been computed for the compressibility equations.

** If $\mathbf{E}(z, \bar{z}, t)$ is real, then $p_{2}(z, \bar{z})$ vanishes identically and we have to change the numeration of the $\mathrm{p}_{v}(z, \bar{z})$ accordingly. The proof that (7.1) where $f$ is an arbitrary function of a complex variable is a solution of (1.1) is given in [1] $\$ 1$. Substituting $Q^{(p)}$ instead of $\phi^{(2 p)} / z^{p}$ into (1.16) of [1] p. 1174 and integrating with respect to $\bar{z}$ we obtain (3.3). (Note that there are some misprints in [1] which are indicated in the Trans. of the Amer. Math. Soc. vol. 57, p. 311, Footnote 15.)

$\dagger$ Unless otherwise indicated the arguments of the functions $Q^{(p)}, F, A$, etc., are $(z, \bar{z})$. 


$$
\left.\begin{array}{rl}
Q^{(1)}=-2 \int_{0}^{z} \text { Fid } \bar{z}, & \\
Q^{(p)}=-\frac{2}{2 p-1}\left[Q_{z}^{(p-1)}-Q_{z}^{(p-1)}(z, 0)+\int_{0}^{\bar{z}}\left(D Q_{\bar{z}}^{(p-1)}+F Q^{(p-1)}\right) d \bar{z}\right], \\
(p=2,3,4, \cdots) .
\end{array}\right\}
$$

$$
Q^{(p)}(z, 0)=0, \quad(p=1,2,3, \cdots),
$$

where

$$
D=-\int_{0}^{\bar{z}} A_{z} d \bar{z}+B, \quad F=-A B-A_{z}+C
$$

If the coefficients $A, B, C$ of (2.1) are polynomials

$$
A=\sum_{m=0}^{N} \sum_{n=0}^{N} a_{m, n} z^{m \bar{Z}^{n}}, \quad B=\sum_{m=0}^{N} \sum_{n=0}^{N} b_{m, n} z^{m \bar{z}^{n}}, \quad C=\sum_{m=0}^{N} \sum_{n=0}^{N} c_{m, n} z^{m} \bar{z}^{n},
$$

then for the coefficients $d_{m, n}$ and $f_{m, n}$ of the developments

$$
D=\sum_{m=0}^{M} \sum_{n=0}^{M} d_{m, n} z^{m \bar{z}^{n}}, \quad F=\sum_{m=0}^{M} \sum_{n=0}^{M} f_{m, n} z^{m} \bar{z}^{n},
$$

we obtain the relations

$$
\begin{aligned}
& d_{0, n}=b_{0, n}, \quad d_{m, n}=-\frac{(m+1) a_{m+1, n-1}}{n}+b_{m, n}, \\
& f_{m, n}=-\left[(m+1) a_{m+1, n}+\sum_{i=0}^{i=m} \sum_{j=0}^{j=n} a_{m-i, n-j} b_{i, j}\right]+c_{m, n} .
\end{aligned}
$$

4. Determination of the $Q^{(p)}$. Since the $A, B, C$ (see (2.1)) are assumed to be polynomials, $D$ and $F$ are also polynomials in $z$, $\bar{z}$, as is indicated in (3.6). (Both $F$ and $D$ are assumed to be of the same degree; this is always permissible, since some $f$ 's or $d$ 's can be assumed to equal zero.) We now write*

$$
Q^{(p-1)}=\sum_{i} \sum_{j} q_{i, j}^{(p-1)} z^{i} \bar{z}^{j}, \quad q_{i, 0}^{(p-1)}=0,
$$

and proceed to the representation of $q_{m, n}^{(p)}$ in terms of the $q_{n, j}^{(p-1)}$. Formal computations yield

$$
Q_{\bar{z}}^{(p-1)}=\sum_{i} \sum_{j} j q_{i, j}^{(p-1) z^{i} \bar{z}^{j-1}}=\sum_{i} \sum_{j}(j+1) q_{i, j+1}^{(p-1) z^{i} \bar{z}^{j}}
$$

* The relations $q_{i, 0}^{(p-1)}=0$ follow from the equation $Q^{(p-1)}(z, 0)=0$. See (3.3). Note that if one of the subscripts $m, n$ of $d_{m, n}$ becomes negative or larger than $M$, it is necessary to substitute 0 for the corresponding $d_{m, n}$. The same holds for $f_{m, n}$. 


$$
\begin{aligned}
& F Q^{(p-1)}=\left[\sum_{\nu} z^{\nu}\left(f_{\nu, 1}+f_{\nu, 1} \bar{z}+f_{\nu, 2} \bar{z}^{2}+\cdots\right)\right]\left[\sum z^{i}\left(q_{i, 1} \bar{z}+q_{i, 2} \bar{z}^{2}+\cdots\right)\right] \\
& =\sum_{m} \sum_{n}\left(\sum_{i=1}^{m} \sum_{j=1}^{n} f_{m-i, n-j}^{(p-1)} q_{i, j}\right) z^{m} \bar{z}^{n} \\
& D Q_{z}^{(n-1)}=\left[\sum_{\nu} z^{\nu}\left(d_{\nu, 0}+d_{\nu, 1} \bar{z}+d_{\nu, 2} \bar{z}^{2}+\cdots\right)\right]\left[\sum_{i} z^{i}\left(q_{i, 1}+2 q_{i, 2} \bar{z}+3 q_{i, 3} \bar{z}^{2}+\cdots\right)\right] \\
& =\sum_{m} \sum_{n}\left(\sum_{i=0}^{m} \sum_{j=0}^{n}(j+1) d_{m-i, n-j} q_{i, j+1}^{(p-1)}\right) z^{m \bar{z}^{n}} \\
& =\sum_{m} \sum_{n}\left(\sum_{i=0}^{m} \sum_{j=1}^{n+1} j d_{m-1, n-j, 1} q_{i, j}^{(p-1)}\right) z^{m} \bar{z}^{n} \\
& F Q^{(p-1)}+D Q_{\bar{z}}^{(p-1)}=\sum_{m} z^{m} \sum_{i=0}^{m}\left\{d_{m-1,0} q_{i, 1}+\left[2 d_{m-i, 0} q_{i, 2}+\left(d_{m-1,1}+f_{m-i, 0}\right) q_{i 1} \bar{z}\right]\right. \\
& \left.+\left[3 d_{m-i, 0} q_{i, 3}+\left(2 d_{m-i, 1}+f_{m-i, 0}\right) q_{i, 2}+\left(d_{m-i, 2}+f_{m-i, 1}\right) q_{i, 1}\right] \bar{z}^{2}+\cdots\right\} \\
& =\sum_{m} \sum_{n}\left[\sum_{i=0}^{m} \sum_{j=1}^{n}\left(j d_{m-i, n-j+1}+f_{m-i, n-j}\right) q_{i, j}^{(p-1)}+(n+1) \sum_{i=0}^{m} d_{m-i, 0} q_{i, n+1}^{(p-1)}\right] z^{m} \bar{z}^{n} \text {. }
\end{aligned}
$$

Therefore

$$
\left.\begin{array}{rl}
q_{m, k}^{(1)}= & -\frac{2}{k} f_{m, k-1}, \\
q_{m, 1}^{(p)}= & -\frac{2}{2 p-1}\left[\sum_{i=0}^{m} d_{m-i, 0} q_{i, 1}^{(p-1)}+(m+1) q_{m+1,1}^{(p-1)}\right], \quad(p=2,3, \cdots), \\
q_{m, n}^{(p)}= & -\frac{2}{2 p-1}\left[\frac{1}{n} \sum_{i=0}^{m} \sum_{k=1}^{n-1}\left(k d_{m-i, n-k}+f_{m-i, n-k-1}\right) q_{i, k}^{(p-1)} .\right. \\
& \left.+\sum_{i=n}^{m}\left(d_{m-i, n} q_{i, n}^{(p-1)}+(m+1) q_{m+1, n}^{(p-1)}\right)\right], \\
\quad(m=0,1,2, \cdots, n=2,3,4, \cdots, p=2,3, \cdots) .
\end{array}\right\}
$$

5. Evaluation of the $q_{m, n}^{(p)}$. If we assume that the $d_{m, n}$ and $f_{m, n}$ are determined, the evaluation of the $q_{m, n}^{(p)}$ represents a considerable computational task. Merely writing down the various pairs which have to be multiplied together and adding the resulting products is quite laborious. Therefore, it is convenient to organize the determination of the $q_{m, n}^{(p)}$ in such manner that one can see automatically what quantities have to be multiplied and added. After such a procedure has been developed it can be conveniently employed for performing the computation on punch card machines.

We shall describe such a procedure, assuming for the sake of simplicity that $M$ in (4.1) is a given number, namely $M=3$. The changes that have to be made when $M$ assumes other values are in many instances clear. If however this is not the case, then we shall write these formulae out explicitly for the general case, i.e., retaining $M$. The expressions $q_{m, n}^{(p)}(p>1)$ to be evaluated are given in (4.5) in the form of sums of 
products of two sets of numbers; the first set are the coefficients of $q_{i, k}^{(\prime)}{ }^{\prime \prime}$, and the second set are $q_{i, i}^{(p-1)}$. This suggests the construction of a set of cards and stencils. The coefficients of $q_{i, i}^{(p-1)}$ are entered on the cards. The stencils arc cards with holes cut in them, and the quantities $q_{i, k}^{(p-1)}$ are entered on the stencils in such a way that when a stencil is placed over a card properly, quantities on the cards appear in the holes in the stencil beside the $q_{i, k}^{(p-1)}$ by which they are to be multiplied.

A card is constructed for each value of $n$; these cards are called the $[\mathrm{S}, n]$ cards. A stencil is constructed for each value of $p$; these stencils are called the $[Q, p]$ stencils. In order to compute $q_{m, n}^{(p)}$ and hence construct the $[\mathbf{Q}, p]$ stencil, we require the $[\mathbf{Q}, p-1]$ stencil and the $[\mathbf{S}, n]$ cards corresponding to* $n=1,2, \cdots, p(M+1)$.

Each $[\mathbf{S}, n]$ card contains a matrix $\left\{s_{r, k}^{(n)}\right\}$ of $M+2$ rows and $M+1$ columns; $r$ indicates the row and $k$ the column. Also when $M=3$,

$$
s_{r, k}^{(n)}=\frac{1}{n}\left[(n+r-5) d_{4-k, 5-r}+f_{4-k, 4-r}\right] .
$$

\begin{tabular}{|c|c|c|c|}
\hline$f_{3.3} / n$ & $s_{1,2}^{(n)}=$ & $f_{1,3} / n$ & $s_{1,4}^{(n)}=$ \\
\hline$s_{2,1}^{(n)}=\left[(n-3) d_{3,3}+f_{3,2}\right] / n$. & $s_{2,2}^{(n)}=\left[(n-3) d_{2,3}+f_{2,2}\right] / n$ & $s_{2,3}^{(n)}=\left[(n-3) d_{1,3}+f_{1,2}\right] / n$ & $s_{2.4}^{(n)}=\left[(n-3) d_{0.3}+f_{0.2}\right] / n$ \\
\hline$s_{3,1}^{(n)}=\left[(n-2) d_{3,2}+f_{3,1}\right] / n$ & $s_{3,2}^{(n)}=\left[(n-2) d_{2,2}+f_{2,1}\right] / n$ & $s_{3,3}^{(n)}=\left[(n-2) d_{1,2}+f_{1,1}\right] / n$ & $s_{3,4}^{(n)}=\left[(n-2) d_{0,2}+f_{0,1}\right] / n$ \\
\hline$s_{4,1}^{(n)}=\left[(n-1) d_{3,1}+f_{3,0}\right] / n$ & $s_{4,2}^{(n)}=\left[(n-1) d_{2,1}+f_{2,0}\right] / n$ & $s_{4,3}^{(n)}=\left[(n-1) d_{1,1}+f_{1,0}\right] / n$ & $s_{4,4}^{(n)}=\left[(n-1) d_{0,1}+f_{0,0}\right] / n$ \\
\hline$s_{5,1}^{(n)}=\quad d_{3,0}$ & $s_{4,1}^{(n)}=\quad d_{2,0}$ & $s_{5,3}^{(n)}=\quad d_{1,0}$ & $s_{5,4}^{(n)}=\quad d_{0,0}$ \\
\hline
\end{tabular}

Fig. 1. A $[\mathrm{S}, n] \operatorname{card}^{* *}$ in the case $M=3$.

For $n \leqq M+1$ (i.e., in our case for $n \leqq 4$ ), the elements of the first $M+2-n$ lines are to be set equal to zero, i.e., in our case $s_{k r}^{(n)}=0$ for $k \leqq 5-n$.

A $[Q, p]$ stencil consists of $(p+1)(M+1)-p$ columns and $(p+1)(M+1)$ rows. The $q_{m, n}^{(p)}$ in the last $M$ columns and $M+1$ last lines are equal to zero. In the case $M=3$, typical stencils are as shown in Figs. 2 and 3 (toe interiors of the rectangles should be cut out).

The number of remaining columns in any stencil will be three greater and the number of rows four greater than in the stencil for the preceding value of $p$. The $[Q, 1]$ stencil has 7 columns, 7 "deleted" columns, and 8 rows; hence the $[Q, 2]$ stencil will have 10 columns, 10 "deleted" columns, and 12 rows; etc. (The $[\mathbf{Q}, 1]$ stencil has 7 columns ( +7 "deleted" columns) and 8 rows and the S cards have 4 columns and 5 rows.) See Fig. 2.

Each member $q_{m, n}^{(p)}$ on the $[\mathbf{Q}, p]$ stencils is specified by three numbers, one superscript and two subscripts. The superscript remains constant for each $\mathbf{Q}$ stencil, i.e., all numbers computed for the $[\mathbf{Q}, 2]$ stencil will have the form $q_{m, n}^{(2)}$, for the $[\mathbf{Q}, 3]$ stencil $q_{m, n}^{(3)}$, etc. The subscripts give the position of the number on the paper, indicating column and row respectively; $q_{4,3}^{(1)}$ means that the number is on the $[\mathbf{Q}, 1]$ stencil in the fourth column and third row; $q_{5,2}^{(2)}$ that the number is on the $[\mathbf{Q}, 2]$ stencil in the fifth column and second row; etc.

* We note that in increasing $p$ to $p+1$ the number of $[S, n]$ cards increases to $(p+1)(M+1)$; however $p(M+1)$ of these cards are exactly the same cards used at the $p$ th stage, so that it is necessary to prepare only $M+1$ new [S, $n$ ] cards.

${ }^{* *}$ The numbers on the $S$ stencil are the coefficients of $q_{i, k}^{(p-1)}, q_{i, 8}^{(p-1)}$ in (4.5). 


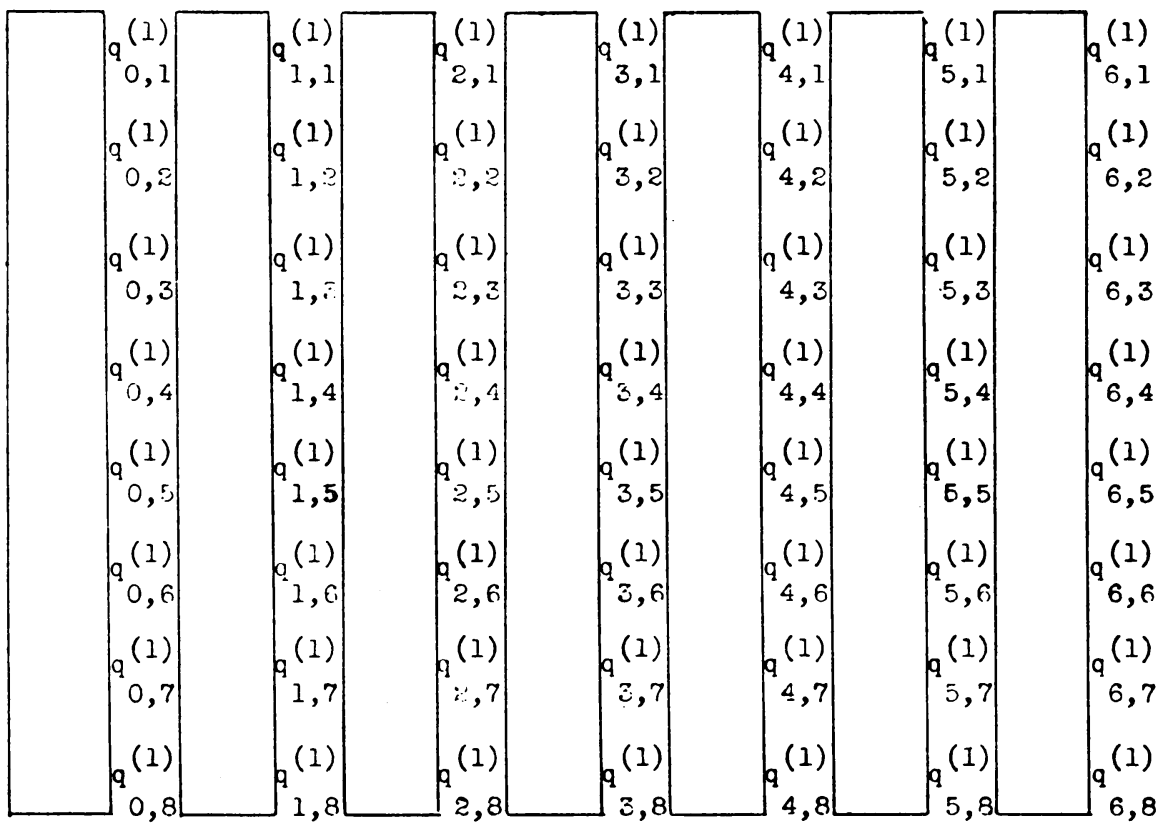

FIG. 2. Stencil $[Q, 1]$.

Using (4.4), we determine $q_{m, p}^{(1)},(m=0,1,2,3, p=1,2,3,4) ; q_{m, p}^{(1)}(m=4,5,6$ or $p=5,6,7,8)$ we set equal to zero. Thus the stencil $[Q, 1]$ has the appearance indicated in Fig. 2. The stencil $[Q, 2]$ consists of 10 columns and 12 rows (besides the deleted columns, i.e. the holes in the stencils). See Fig. 3. We now proceed to the computation of the $[Q, 2]$ stencil. To compute $q_{m, n}^{(2)}$ we proceed as follows: the second subscript $(m, n)$ indicates the $q_{m, n}^{(1)}$ on which we must fix our attention. Since there are four more rows and three more columns on the $[Q, 2]$ stencil than on the $[Q, 1]$ stencil, there will be no corresponding $q_{m, n}^{(1)}$ for these last columns and rows-we fill in these last 3 columns and last four rows on the $[Q, 2]$ stencil with zeros. To construct the remainder of the $[Q, 2]$ stencil, we place the $[Q, 1]$ stencil on top of the $[\mathrm{S}, n]$ card so that the number $s_{M+2, M+1}^{(n)}$ on the $[\mathbf{S}, n]$ card occupies the space to the left of $q_{m, n}^{(1)} *^{*}$ There are then 20 numbers on the $\mathbf{S}$ card adjacent (on the left) to 20 numbers on the $[Q, 1]$ stencil, which indicates the products $(20$ in all) which have to be obtained; once the products are computed they are to be summed.

In this manner we may tentatively fill in the whole $[Q, 2]$ stencil. Now we compute the product of $(m+1) q_{m+1, n}^{(1)}$ and add this to each of the "tentative $q_{m, n}^{(2)}$." If this number is multiplied by $-2 / 3$ [in the case of an arbitrary $p$ by $-2 /(2 p-1)$ ], we obtain the "final $q_{m, n}^{(2)}$ " of the stencil $[\mathbf{Q}, 2]$.

Having completed the $[Q, 2]$ stencil we may repeat the above operations but with $[\mathbf{Q}, 2]$ and $q_{m, n}^{(2)}$ replacing $[\mathbf{Q}, 1]$ and $q_{m, n}^{(1)}$, respectively, to compute the $[\mathbf{Q}, 3]$ stencil. Similarly, we can compute as many $\mathbf{Q}$ stencils as desired.

* See Fig. 4 , where the $[Q, 1]$ stencil covers the $[S, 4]$ card, and the arrangement for the computation of $q_{8.4}^{(2)}$ is indicated. 


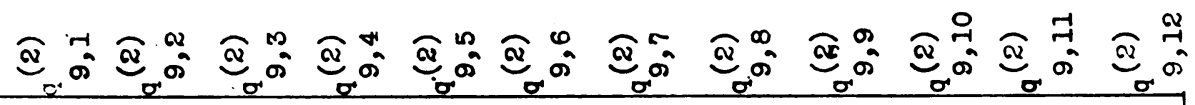

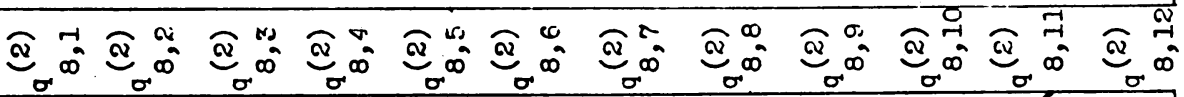

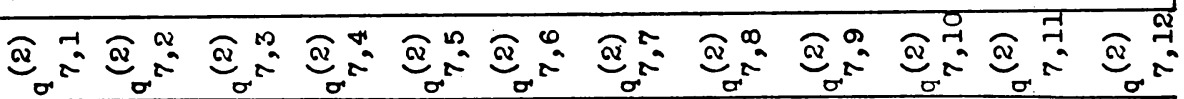

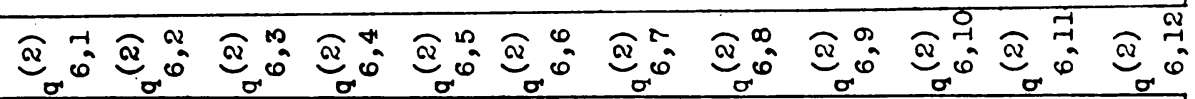

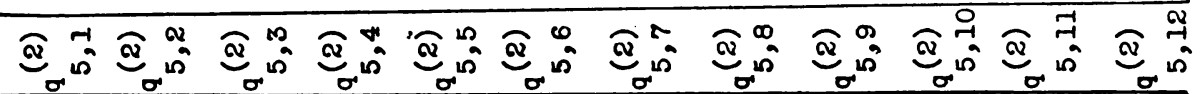

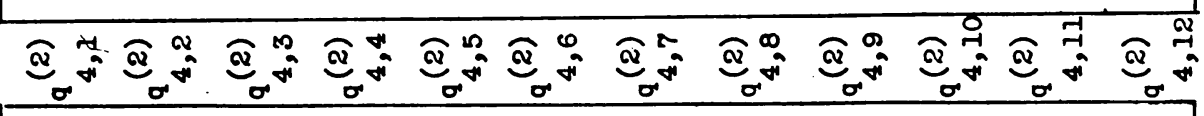

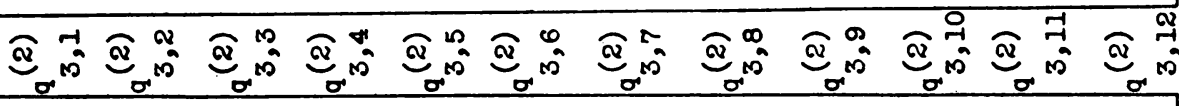

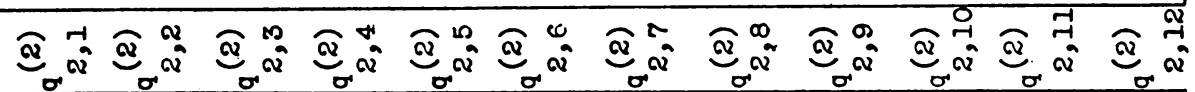

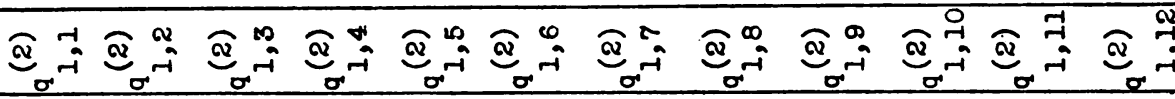

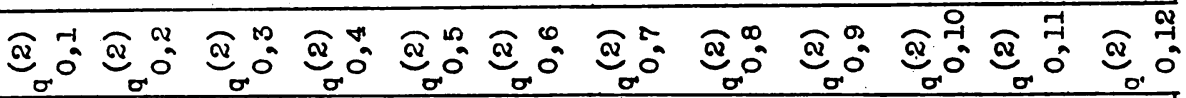




$\begin{array}{llll}s_{11}^{(5)} & s_{12}^{(5)} & s_{13}^{(5)} & s_{14}^{(5)} \\ s_{21}^{(5)} & s_{22}^{(5)} & s_{23}^{(5)} & s_{24}^{(5)} \\ s_{31}^{(5)} & s_{32}^{(5)} & s_{33}^{(5)} & s_{34}^{(5)} \\ s_{41}^{(5)} & s_{42}^{(5)} & s_{43}^{(5)} & s_{44}^{(5)} \\ s_{51}^{(5)} & s_{52}^{(5)} & s_{53}^{(5)} & s_{54}^{(5)}\end{array}$

FIG. 4. [S, 5] card.

6. Example. In this section, we shall illustrate our general descritpion by a specific example. We shall indicate the operations to be performed on punch card machines only. The arrangements and the methods of how the computation is to be performed on these machines can be found in books on punch card methods e.g. in [3]. More specifically we refer for operations concerning complex numbers to [4], and for computations used in the present paper to [5].

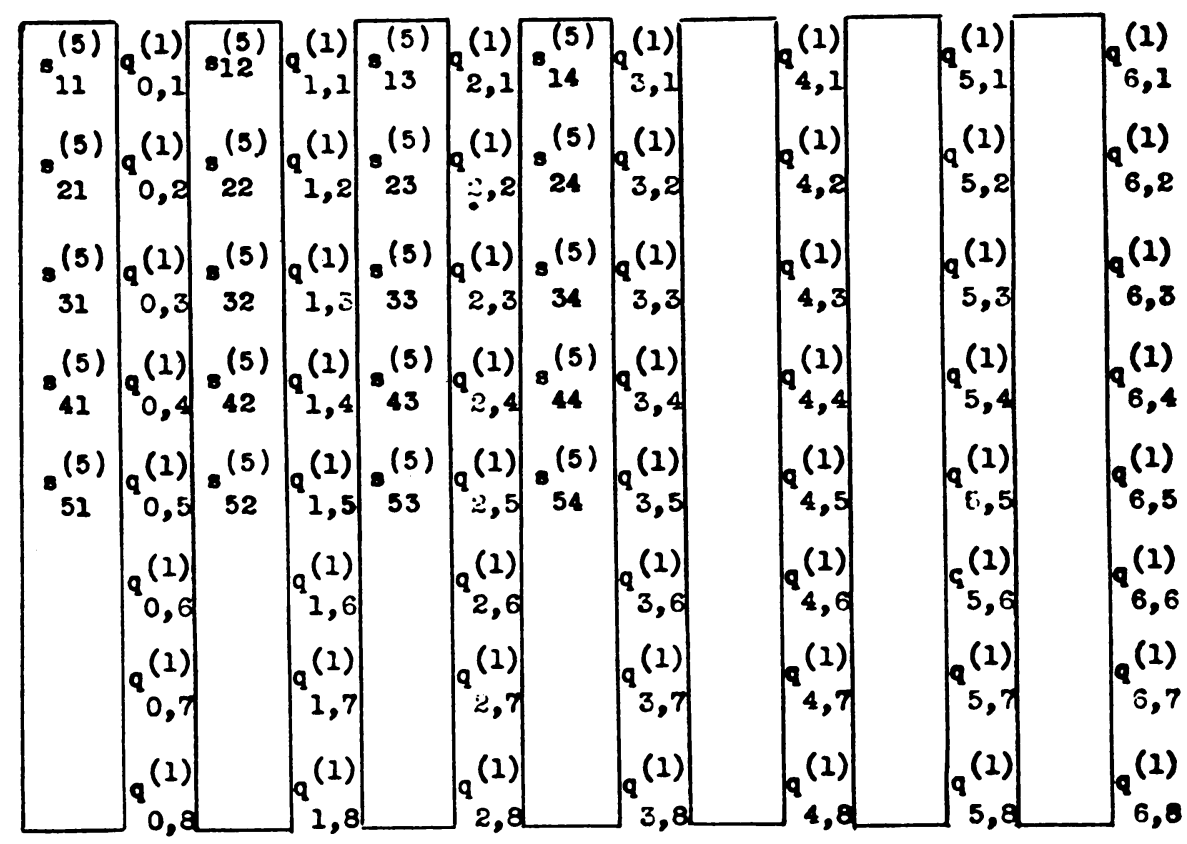

FIG. 5. When stencil $[Q, 1]$ is placed on the $[S, 5]$ card in order to compute $q_{3, b}^{(2)}$, the $s_{m, r}^{(6)}$ appcar as indicated above. $\sum_{n} \sum_{m} s_{m, n+1}^{(5)} q_{n, m}^{(1)}$ gives the "tentative $q_{3.5}^{(2)}$ "

As an illustration of the above method, we now give $a_{m, n}, b_{m, n}, c_{m, n}$ the specific values,

$$
\begin{aligned}
& a_{0,0}=1, \quad a_{m, n}=\frac{2 m n+i\left(m^{2}-n^{2}\right)}{\left(m^{2}+n^{2}\right)}, \\
& b_{0,0}=1, \quad b_{m, n}=\frac{1}{\left(m^{2}+n^{2}\right)},
\end{aligned}
$$




$$
c_{0, n}=1, \quad i_{m, n}=\frac{(m+n)+i(m-n)}{\left(m^{2}+n^{2}\right)} .
$$

In Table 1 the values of $d_{m, n}$ and $f_{m, n}$ which have been determined according to (3.7) and (3.8) are tabulated for $m=0,1,2,3,4, n=0,1,2,3,4$.

TABLE 1. The values of $d_{m, n}$ and $f_{m, n}$.

\begin{tabular}{c|c|r|r|r|r}
\hline$m$ & $n$ & $\operatorname{Re}\left(d_{m, n}\right)$ & $\operatorname{Im}\left(d_{m, n}\right)$ & $\operatorname{Re}\left(f_{m, n}\right)$ & \multicolumn{1}{c}{$\operatorname{In}\left(f_{m, n}\right)$} \\
\hline 0 & 0 & 1.0000 & 0.0000 & 0.0000 & -1.0000 \\
& 1 & 1.0000 & 0.0000 & -1.0000 & 0.0000 \\
& 2 & 0.2500 & 0.0000 & -0.5500 & 2.1000 \\
& 3 & 0.1111 & 0.0000 & -0.3778 & 2.7167 \\
\hline 1 & 0 & 1.0000 & 0.0000 & 0.0000 & -2.0000 \\
& 1 & 0.0000 & -2.0000 & -2.1000 & -1.2000 \\
& 2 & -0.8000 & -0.6000 & -3.4000 & 1.6500 \\
& 3 & -0.6667 & 0.0000 & -3.1962 & 3.5581 \\
\hline 2 & 0 & 1.0000 & 0.0000 & 0.2500 & -4.5000 \\
& 1 & 0.0000 & -3.2000 & -3.2000 & -4.0550 \\
& 2 & -0.9000 & -1.2000 & -5.4942 & -1.1538 \\
& 3 & -0.9231 & -0.3846 & -6.0154 & 1.6216 \\
\hline 3 & 0 & 1.0000 & 0.0000 & 0.2222 & -5.9157 \\
& 1 & 0.0000 & -4.0000 & -3.2324 & -6.3183 \\
& 2 & -0.9412 & -1.7647 & -6.2154 & -4.0216 \\
& 3 & -1.0667 & -0.8000 & -7.6534 & -1.1200 \\
\hline
\end{tabular}

To determine $q_{m, n}^{(2)}$ by use of punch cards we proceed as follows: for each number on an $\mathbf{S}$ card, we make one punch-card, and separate into groups. Thus, in order to compute the $[Q, 2]$ stencil which has 8 rows we would need 8 groups of $S$ cards. See Table 2. In the group [S, 1] there would be four cards, in [S, 2] eight cards, in [S, 3] twelve cards, in [S, 4] sixteen cards, in [S, 5] and in all succeeding groups twenty cards.

We arrange the numbers in the first line of the $[Q, 1]$ stencil (see Table 3 ) as follows:

$\begin{array}{llll}q_{0,1}^{(1)} & 0 & 0 & 0 \\ q_{1,1}^{(1)} & q_{0,1}^{(1)} & 0 & 0 \\ q_{2,1}^{(1)} & q_{1,1}^{(1)} & q_{0,1}^{(1)} & 0 \\ q_{3,1}^{(1)} & q_{2,1}^{(1)} & q_{1,1}^{(1)} & q_{0,1}^{(1)} \\ q_{4,1}^{(1)} & q_{3,1}^{(1)} & q_{2,1}^{(1)} & q_{1,1}^{(1)} \\ q_{5,1}^{(1)} & q_{4,1}^{(1)} & q_{3,1}^{(1)} & c_{2,1}^{(1)} \\ q_{6.1}^{(1)} & q_{5,1}^{(1)} & q_{4,1}^{(1)} & q_{3,1}^{(1)}\end{array}$

For each of these we punch one card and separate into groups as indicated. We do the same for each row of the $[\mathbf{Q}, 1]$ stencil, so that we will have seven such groups in total. (There are then four $q_{0,1}^{(1)}, q_{1,1}^{(1)}, \ldots$ cards and a total of six zero cards.) 
TARLI: 2. The values of $s_{i, j}^{(n,}$, for $n=1,2,3,4,5$.*

\begin{tabular}{|c|c|c|c|c|c|c|c|c|c|}
\hline$n$ & $i$ & $j$ & $\operatorname{Re}\left(s_{i, j}^{(n)}\right)$ & $\operatorname{lm}\left(s_{i, j}^{(n)}\right)$ & $n$ & $i$ & $j$ & $\operatorname{Re}\left(s_{i, j}^{(n)}\right)$ & $\left(\operatorname{Im}\left(s_{i, j}^{(n)}\right)\right.$ \\
\hline \multirow[t]{4}{*}{1} & 5 & 4 & 1.0000 & 0.0000 & \multirow[t]{13}{*}{4} & 5 & 3 & 1.0000 & 0.0000 \\
\hline & 5 & 3 & 1.0000 & 0.0000 & & 4 & 3 & 0.0000 & -2.0000 \\
\hline & 5 & 2 & 1.0000 & 0.0000 & & 3 & 3 & -0.9250 & -0.6000 \\
\hline & 5 & 1 & 1.0000 & 0.0000 & & 2 & 3 & -1.0167 & 0.4125 \\
\hline \multirow{9}{*}{2} & & & & & & 5 & 2 & 1.0000 & 0.0000 \\
\hline & 5 & 4 & 1.0000 & 0.0000 & & 4 & 2 & 0.0625 & -3.3750 \\
\hline & 4 & 4 & 0.5000 & -0.5000 & & 3 & 2 & -1.2500 & -1.6125 \\
\hline & 5 & 3 & 1.0000 & 0.0000 & & 2 & 2 & -1.6043 & -0.3846 \\
\hline & 4 & 3 & 0.0000 & -2.0000 & & 5 & 1 & 1.0000 & 0.0000 \\
\hline & 5 & 2 & 1.0000 & 0.0000 & & 4 & 1 & 0.0555 & -4.4792 \\
\hline & 4 & 2 & 0.1250 & -3.7500 & & 3 & 1 & -1.2787 & -2.4619 \\
\hline & 5 & 1 & 1.0000 & 0.0000 & & 2 & 1 & -1.8205 & -1.2054 \\
\hline & 4 & 1 & 0.1111 & -4.9583 & & & & & \\
\hline \multirow[t]{13}{*}{3} & 5 & 4 & 1.0000 & 0.0000 & \multirow{19}{*}{5} & $\begin{array}{l}5 \\
4\end{array}$ & $\begin{array}{l}4 \\
4\end{array}$ & $\begin{array}{l}1.0000 \\
0.8000\end{array}$ & $\begin{array}{r}0.0000 \\
-0.2000\end{array}$ \\
\hline & 4 & 4 & 0.6667 & -0.3333 & & 3 & 4 & -0.0500 & 0.0000 \\
\hline & 3 & 4 & -0.2500 & 0.0000 & & 2 & 4 & -0.0655 & 0.4200 \\
\hline & 5 & 3 & 1.0000 & 0.0000 & & 1 & 4 & -0.0755 & 0.5433 \\
\hline & 4 & 3 & 0.0000 & -2.0000 & & 5 & 3 & 1.0000 & 0.0000 \\
\hline & 3 & 3 & -0.9667 & -0.6000 & & 4 & 3 & 0.0000 & -2.0000 \\
\hline & 5 & 2 & 1.0000 & 0.0000 & & 3 & 3 & -0.9000 & -0.6000 \\
\hline & 4 & 2 & 0.0833 & -3.5000 & & 2 & 3 & -0.9467 & 0.3300 \\
\hline & 3 & 2 & -1.3667 & -1.7500 & & 1 & 3 & -0.6392 & 0.7116 \\
\hline & 5 & 1 & 1.0000 & 0.0000 & & 5 & 2 & 1.0000 & 0.0000 \\
\hline & 4 & 1 & 0.0741 & -4.6389 & & 4 & 2 & 0.5000 & -3.3000 \\
\hline & 3 & 1 & -1.3912 & -2.6943 & & 3 & 2 & -1.1800 & -1.5300 \\
\hline & & & & & & 2 & 2 & -1.4681 & -0.3846 \\
\hline \multirow[t]{6}{*}{4} & 5 & 4 & 1.0000 & 0.0000 & & 1 & 2 & -1.2031 & 0.3243 \\
\hline & 4 & 4 & 0.7500 & -0.2500 & & 5 & 1 & 1.0000 & 0.0000 \\
\hline & 3 & 4 & -0.1250 & 0.0000 & & 4 & 1 & 0.0444 & -4.3833 \\
\hline & 2 & 4 & -0.1097 & 0.5250 & & 3 & 1 & -1.2112 & -2.3225 \\
\hline & & & & & & 2 & 1 & -1.6697 & -1.1243 \\
\hline & & & & & & 1 & 1 & -1.5307 & -0.2240 \\
\hline
\end{tabular}

TABLE 3. The values of $q_{m, k}^{(1)}$.

\begin{tabular}{|c|c|c|c|c|c|c|c|}
\hline$m$ & $k$ & $\operatorname{Re}\left(q_{m, k}^{(1)}\right)$ & $\operatorname{Im}\left(q_{m, k}^{(1)}\right)$ & $m$ & $k$ & $\operatorname{Re}\left(q_{m, k}^{(1)}\right)$ & $\operatorname{Im}\left(q_{m, k}^{(1)}\right)$ \\
\hline \multirow[t]{4}{*}{0} & 1 & 0.0000 & 2.0000 & 2 & 1 & -0.5000 & 9.0000 \\
\hline & 2 & 1.0000 & 0.0000 & & 2 & 3.2000 & 4.0500 \\
\hline & 3 & 0.3666 & -1.4000 & & 3 & 3.6628 & 0.7692 \\
\hline & 4 & 0.1889 & -1.3583 & & 4 & 3.0077 & -0.8108 \\
\hline \multirow[t]{4}{*}{1} & 1 & 0.0000 & 4.0000 & 3 & 1 & -0.4444 & 11.8333 \\
\hline & 2 & 2.1000 & 1.2000 & & 2 & 3.2324 & 6.3183 \\
\hline & 3 & 2.2667 & -1.1000 & & 3 & 4,1436 & 2.6810 \\
\hline & 4 & 1.5981 & -1.7791 & & 4 & 3.8267 & 0.5600 \\
\hline
\end{tabular}

* The $s_{i, j}^{(n)}$, for $n=6,7,8$ have to be computed in a similar manner. 
Starting with the group of cards for line one, we take the "dot" product for each sub-group with the $[\mathbf{S}, 1]$ cards. ${ }^{*}$ Let the numbers in the first row of the $[\mathbf{S}, 1]$ card be denoted by $s_{51}^{(1)}, s_{52}^{(1)}, s_{53}^{(1)}, s_{54}^{(1)}$; we then have to compute the following products:

$$
\begin{aligned}
& q_{0,1}^{(1)} \cdot s_{5,4}^{(1)}+0 \cdot s_{5,3}^{(1)}+0 \cdot s_{5,2}^{(1)}+0 \cdot s_{5,1}^{(1)} \\
& q_{1,1}^{(1)} \cdot s_{5,4}^{(1)}+q_{0,1}^{(1)} \cdot s_{5,3}^{(1)}+0 \cdot s_{5,2}^{(1)}+0 \cdot s_{5,1}^{(1)} \\
& q_{1,1}^{(1)} \cdot s_{5,4}^{(1)}+q_{1,1}^{(1)} \cdot s_{5,3}^{(1)}+q_{0,1}^{(1)} \cdot s_{5,2}^{(1)}+0 \cdot s_{5,1}^{(1)} \\
& q_{3,1}^{(1)} \cdot s_{5,4}^{(1)}+q_{2,1}^{(1)} \cdot s_{5,3}^{(1)}+q_{1,1}^{(1)} \cdot s_{5,2}^{(1)}+q_{0,1}^{(1)} \cdot s_{5,1}^{(1)} \\
& \xi_{1,1}^{(1)} \cdot s_{5,4}^{(1)}+q_{3,1}^{(1)} \cdot s_{5,3}^{(1)}+q_{2,1}^{(1)} \cdot s_{5,2}^{(1)}+q_{1,1}^{(1)} \cdot s_{5,1}^{(1)}
\end{aligned}
$$

The actual procedure is to form the products $q_{0,1}^{(1)} \cdot s_{5,4}^{(1)}, q_{1,1}^{(1)} \cdot s_{5,4}^{(1)}, q_{2,1}^{(1)} \cdot s_{5,4}^{(1)}, \ldots$; $0 \cdot s_{5,3}^{(1)}, q_{0,1}^{(1)} \cdot s_{5,3}^{(1)}, q_{1,1}^{(1)} \cdot s_{5,3}^{(1)}$ and enter these together with appropriate signs etc. on the corresponding cards. These cards are then assorted in sub-groups as indicated and the products summed to yield the desired "dot-product."

To compute the numbers which are to appear in the second line of the $[Q, 2]$ stencil, the numbers in the second line of the $[Q, 1]$ stencil are arranged as follows:

$\begin{array}{cccc}q_{0,2}^{(1)} & 0 & 0 & 0 \\ q_{1,2}^{(1)} & q_{0,2}^{(1)} & 0 & 0 \\ q_{2,2}^{(1)} & q_{1,2}^{(1)} & q_{0,2}^{(1)} & 0 \\ q_{3,2}^{(1)} & q_{2,2}^{(1)} & g_{1,2}^{(1)} & q_{0,2}^{(1)} \\ \cdot & \cdot & \cdot & \cdot \\ q_{\mathbf{8}, 2}^{(1)} & q_{5,2}^{(1)} & q_{\mathbf{1}, 2}^{(1)} & q_{3,2}^{(1)}\end{array}$

For each of these numbers one card is punched and separated into groups of eight cards as indicated below, using the cards previously punched for the first line of the $[Q, 1]$ stencil:

$\begin{array}{cccccccc}q_{0,2}^{(1)} & q_{0,1}^{(1)} & 0 & 0 & 0 & 0 & 0 & 0 \\ q_{1,2}^{(1)} & q_{1,1}^{(1)} & q_{0,2}^{(1)} & q_{0,1}^{(1)} & 0 & 0 & 0 & 0 \\ q_{2,2}^{(1)} & q_{2,1}^{(1)} & q_{1,2}^{(1)} & q_{1,1}^{(1)} & q_{0,2}^{(1)} & c_{0,1}^{(1)} & 0 & 0 \\ q_{3,2}^{(1)} & q_{3,1}^{(1)} & q_{2,2}^{(1)} & q_{2,1}^{(1)} & q_{1,2}^{(1)} & q_{1,1}^{(1)} & \varsigma_{0,2}^{(1)} & q_{0,1}^{(1)} \\ . & \cdot & . & . & . & . & . & .\end{array}$

The dot product of each of these groups in the [S, 2] cards are then taken. Thus:

$$
\begin{aligned}
& q_{0,2}^{(1)} \cdot s_{5,4}^{(2)}+q_{0,1}^{(1)} \cdot s_{4,4}^{(2)}+0 \cdot s_{5,3}^{(2)}+0 \cdot s_{4,3}^{(2)}+0 \cdot s_{5,2}^{(2)}+0 \cdot s_{4,2}^{(2)}+0 \cdot s_{5,1}^{(2)}+0 \cdot s_{4,1}^{(2)} \\
& q_{3,2}^{(1)} \cdot s_{5,4}^{(2)}+q_{3,1}^{(1)} \cdot s_{4,4}^{(2)}+\epsilon_{2,2}^{(1)} \cdot s_{5,3}^{(2)}+q_{2,1}^{(1)} \cdot s_{4,3}^{(2)}+q_{1,2}^{(1)} \cdot s_{5,2}^{(2)}+q_{1,1}^{(1)} \cdot s_{4,2}^{(2)}+q_{0,2}^{(1)} \cdot s_{5,1}^{(2)}+q_{0,1}^{(1)} \cdot s_{4,1}^{(2)}
\end{aligned}
$$

The actual products are again taken as indicated before, and then reassorted and summed.

This process is repeated for each line of the $[Q, 1]$ stencil until each group has twenty cards in it, after which the first line is discarded when a new line is added so that there are never more than twenty cards. In this way the "tentative $[Q, 2]$ sten-

* Note that, in general, both $q_{m, n}^{(p-1)}$ and $s_{r, \downarrow}^{(n)}$ are complex, and in crder to compute $q_{m, n}^{(p-1)} \cdot s_{r, k}^{(n)}$ on punch card machines we have to use rules for the evaluation of complex numbers. 
cil" is computed and it is necessary only to add $(m+1) q_{m+1, n}^{(1)}$ and multiply the result by $-2 / 3$ to obtain the actual values of the elements of the stencil $[Q, 2]$. This procedure may then be repeated to obtain the values of the elements of the stencils $[\mathbf{Q}, 3]$, (i.e. the quantities $q_{m, p}^{(3)}$ ) $[\mathbf{Q}, 4]$ etc.

In Tables 2 and 3 the values of the elements of the cards $[S, n]$ and those of the stencil $[\mathbf{Q}, 1]$ are given.

7. Conclusion. Application of the differential analyzer to the construction of particular solutions. As soon as a sufficient number of $[Q, p]$ stencils (i.e. $q_{m, n}^{(p)}$ ) have been prepared we can, by (3.1) and (3.2) and standard methods of evaluation of polynomials on punch card machines, determine the values of $p_{2 \nu-1}$ and $p_{2 \nu}$ for a sufficiently dense lattice of points.

The second step, the determination of the coefficients $a_{\nu}$ in the expression (1.2) in order to obtain a solution of (1.1) which assumes the prescribed boundary values, will be discussed in a subsequent paper. The basic idea of the procedure to be employed has already been indicated in [2]; we shall however discuss this in more detail from the point of view of numerical analysis.

Sometimes we need solutions of (1.1) in connection with questions other than the boundary value problem, and it is convenient to apply the method of particular solutions in the following slightly modified form.

As was proved in [1], $\$ 1$,

$$
\zeta(z, \bar{z})=\int_{-1}^{1} \mathrm{E}(z, \bar{z}, t) f\left[(z / 2)\left(1-t^{2}\right)\right] d t /\left(1-t^{2}\right)^{1 / 2},
$$

where $f$ is an arbitrary analytic function of a complex variable, which is regular at the origin, is a solution of (1.1).

There are instances in which a large number of solutions of the same equation are required, and the corresponding functions $f$ are known. (See (7.1).) (This situation occurs, for example, if an "atlas" of solutions has been prepared.) In these cases it is then very convenient to employ graphical methods. In the following we shall indicate two graphical methods for the evaluation of (7.1). Both can be performed conviently by use of a differential analyzer.

I. One prepares once and for all for a given equation (1.1) diagrams in which the curves

$$
\begin{aligned}
& Y=E_{1}\left(z_{\nu}, \bar{z}_{\nu}, t\right)=\operatorname{Re}\left[\mathrm{E}\left(z_{\nu}, \bar{z}_{\nu}, t\right)\right],-1 \leqq t \leqq 1, \quad\left(z_{\nu}, \bar{z}_{\nu}\right) \text { fixed, } \\
& Y=E_{2}\left(z_{\nu}, \bar{z}_{\nu}, t\right)=\operatorname{Im}\left[\mathrm{E}\left(z_{\nu}, \bar{z}_{\nu}, t\right)\right],-1 \leqq t \leqq 1, \quad\left(z_{\nu}, \bar{z}_{\nu}\right) \text { fixed, }
\end{aligned}
$$

for a number of points $(x, y)=\left(x_{\nu}, y_{\nu}\right), \nu=1,2,3, \cdots$ are drawn. Further one has to prepare tables for the values $\frac{1}{2} z_{\nu}\left(1-t_{\mu}^{2}\right)$, for $t_{\mu}=-1,-1+\alpha,-1+2 \alpha, \cdots, 1$, where $\alpha$ is a sufficiently small positive constant. $z_{\nu}=x_{\nu}+\mathrm{i} y_{\nu}$ denote the coordinates of points mentioned above. If now the function $f(z)=u(z, \bar{z})+i v(z, \bar{z})$ is given, say in the form of two diagrams for curves $u(z, \bar{z})=$ const. and $v(z, \bar{z})=$ const., we draw (using the tables mentioned above) the curves

$Y=u\left[z_{v}\left(1-t^{2}\right), \bar{z}_{\nu}\left(1-t^{2}\right)\right], \quad Y=v\left[z_{\nu}\left(1-t^{2}\right), \bar{z}_{\nu}\left(1-t^{2}\right)\right], \quad-1 \leqq t \leqq 1$.

Using these diagrams and those mentioned above and employing a differential analyzer (or simply an integrator) we compute the real part of (7.1), 


$$
\begin{aligned}
\int_{t=-1}^{t=1}\left\{E_{1}\left(z_{\nu}, \bar{z}_{\nu}, t\right) u\left[\frac{1}{2} z_{\nu}\left(1-t^{2}\right), \frac{1}{2} \bar{z}_{\nu}\left(1-t^{2}\right)\right]\right. & \\
& \left.-E_{2}\left(z_{\nu}, \bar{z}_{\nu}, t\right) v\left[\frac{1}{2} z_{\nu}\left(1-t^{2}\right),{ }_{2}^{1} \bar{z}_{\nu}\left(1-t^{2}\right)\right]\right\} d t,
\end{aligned}
$$

and analogously its imaginary part.

II. Sometimes it is not sufficient to determine the values of (7.1) at a set of points $\left(x_{v}, y_{v}\right)$ which are prescribed in advance. Then one can apply the following procedure which was suggested to the author by Mr. Hans Kraft.

One prepares (once and for all) diagrams

$$
E_{1}\left(z, \bar{z}, t_{r}\right)=\text { const., } E_{2}\left(z, \bar{z}, t_{r}\right)=\text { const., } t_{v} \text { const. }
$$

for a set of values $t_{\nu}=-1,-1+\alpha,-1+2 \alpha, \cdots, 1$.

Using these diagrams and the tables (described in method I, for every required value of $z$ we can easily determine the curve (7.3) and evaluate the real and imaginary part of (7.1).

Remark. The procedure I can be performed by the use of punch card machines. In this case instead of diagrams (7.2) it is necessary to prepare master cards.

The author should like to thank Professor George E. Hay for his exceedingly helpful advice and friendly criticism.

\section{BIBLIOGRAPHY}

1. Stefan Bergman, Zur Theorie der Funktionen, die eine lineare partielle Differentialgleichung befriedigen, Recueil Mathématique (Mat. Sbornik) N.S. 2, 1169-1198 (1937).

2. Stefan Bergman, The approximation of functions satisfying a linear partial differential equation, Duke Math. Journal, 6, 537-561 (1940).

3. W. J. Eckert, Punched card methods in scientific computation, Columbia University, 1940.

4. Everett Kimball, Jr., A method of technical computations by punched card equipment, Publication of the Bureau of the Census, Washington, D. C.

5. R. Lorant, Digiting without sorting, I.B.M. Pointers No. 461. 